\title{
Fungicide Resistance Action Committee's (FRAC) Classification Scheme of Fungicides According to Mode of Action 1
}

\section{Frederick M. Fishel ${ }^{2}$}

This guide addresses resistance to pesticides and describes the Fungicide Resistance Action Committee's (FRAC) classification of fungicides and bactericides registered for use in Florida by their modes of action. A cross reference of active ingredient common names with corresponding examples of their trade names is also provided.

Fungicide-resistant plant pathogens are not new. Although the first fungicide resistance confirmation was in 1960, there were few subsequent incidences up until 1970. Since then, there have been more incidences, especially with the introduction of systemic fungicides. Also of concern has been the amount of time taken for resistance to emerge; sometimes within two years of a new commercial fungicide introduction. Fungicide resistance is not unique. Insecticide-resistant insects, herbicide-resistant weeds and antibiotic-resistant bacteria are well documented. These pests have two common traits: they have exceptionally large populations with a rapid rate of reproduction. Weeds were the last category of pests to show resistance because they only reproduce, at most, once per year. Insects reproduce with multiple generations in a single year; some bacteria and fungi reproduce several times in a single hour. Where large populations exist, great genetic diversity exists within the population. Within these populations, there will be several individuals that are tolerant of chemical control measures, perhaps only one in a million or billion. Pests typically become resistant when the same pesticide is used repeatedly within a single year or for several consecutive years. Some researchers believe selection pressure forces pests to mutate. However, there are more likely reasons for resistance:

- There were always a few of the resistant types present.

- When the pesticide is applied, the susceptible types are controlled, and then the smaller, resistant populations increase and re-infest the site.

1. This document is PI-94, one of a series of the Pesticide Information Office, Florida Cooperative Extension Service, Institute of Food and Agricultural Sciences, University of Florida. Original publication date January 2006. Visit the EDIS Web Site at http://edis.ifas.ufl.edu.

2. Frederick M. Fishel, associate professor, Agronomy Department, and Director, Pesticide Information Office; Florida Cooperative Extension Service, Institute of Food and Agricultural Sciences, University of Florida, Gainesville, FL 32611.

The use of trade names in this publication is solely for the purpose of providing specific information. UF/IFAS does not guarantee or warranty the products named, and references to them in this publication does not signify our approval to the exclusion of other products of suitable composition.

The Institute of Food and Agricultural Sciences (IFAS) is an Equal Opportunity Institution authorized to provide research, educational information and other services only to individuals and institutions that function with non-discrimination with respect to race, creed, color, religion, age, disability, sex, sexual orientation, marital status, national origin, political opinions or affiliations. U.S. Department of Agriculture, Cooperative Extension Service, University of Florida, IFAS, Florida A. \& M. University Cooperative Extension Program, and Boards of County Commissioners Cooperating. Larry Arrington, Dean 


\section{Cross Resistance versus Multiple Resistance}

More than 50 different fungicide active ingredients and many more trade products, including mixtures, are available to agricultural producers in Florida today. Many of these active ingredients work in the same way; in other words, they have the same mode of action. Although there are numerous trade products available at the current time, there are fewer than 20 growth mechanisms affected by these fungicides. When a certain plant pathogen is not controlled by fungicides affecting the same growth process, it is said to be cross-resistant. An example of a plant pathogen that has cross resistance is one that is resistant to fungicides in the chemical groups, triazoles and pyrimidines, both which are demethylation inhibitors which disrupt sterol synthesis. A more serious concern is multiple resistance. This is a phenomenon that occurs when a plant pathogen is not controlled by fungicides which affect different plant-growth processes. For example, a plant pathogen that is resistant to fungicides which inhibit both mitosis and protein synthesis, two differing fungal growth processes, would be labeled as a plant pathogen having multiple resistance.

\section{Fungicide Selection}

Farmers and crop advisors need to know which fungicides are best suited to combat resistant plant pathogens. To support the use of fungicides suitable for resistance management, the FRAC numerical classification of fungicides in Table 1 is used on fungicide labeling by some manufacturers. The fungicides are classified according to their modes of action, collective and chemical group names, and active ingredient common names. Some examples of popular trade names are provided in Table 2 as a cross reference. The tables do not include all fungicides that are registered for use globally or in the U.S.; rather, those available only in Florida. Those which have an intrinsic "high risk" of resistance evolution are identified. "High risk" is determined by the following indicators:

- Cross resistance with existing fungicides;
- Laboratory studies have shown resistant mutants within the population;

- The active ingredient is known for the practice of repetitive use or sustained treatments;

- The active ingredient is known to have an extensive area of use; and,

- The target plant pathogens are known to have large populations with rapid multiplication.

The system is encouraged by FRAC for fungicide registrants to indicate the mode of action group in a uniform location on their product labels; some registrants currently identify the group on the front panel of their product labels. Similar systems have been proposed and encouraged for herbicides and insecticides. Because of the great variety of trade names and package mixtures of fungicides, it is difficult for agricultural producers to keep track of which modes of action they use.

\section{Additional Information}

Fungicide Resistance Action Committee (FRAC): http://www.frac.info/

McCoy, C.W., M.E. Rogers, and L.W. Timmer. 2004. 2005 Florida citrus pest management guide: pesticide resistance and resistance management. UF/IFAS EDIS Document ENY-624. http://edis.ifas.ufl.edu/CG026.

Tomlin, C.D.S., ed. 2003. The pesticide manual: a world compendium, $13^{\text {th }}$ edition. The British Crop Protection Council. 1250 pp., ISBN 1901396134. 
Table 1. FRAC's classification of fungicides registered for use in Florida by FRAC numerical code, mode of action, chemical group, and active ingredient common name with high risk indication.

\begin{tabular}{|c|c|c|c|c|c|}
\hline $\begin{array}{l}\text { FRAC } \\
\text { code }^{*}\end{array}$ & Mode of action & Group name & Chemical group & Common name & $\begin{array}{l}\text { Resistance } \\
\text { risk } \\
\text { indication }\end{array}$ \\
\hline 1 & $\begin{array}{l}\text { Inhibition of mitosis } \\
\text { and cell division }\end{array}$ & MBC fungicides & $\begin{array}{l}\text { Benzimidazoles } \\
\text { Thiophanates }\end{array}$ & $\begin{array}{l}\text { Carbendazim } \\
\text { Thiabendazole } \\
\text { Thiophanate-methyl }\end{array}$ & HIGH RISK \\
\hline 2 & $\begin{array}{l}\text { Inhibition of lipids and } \\
\text { membrane synthesis }\end{array}$ & Dicarboximides & & $\begin{array}{l}\text { Iprodione } \\
\text { Vinclozolin }\end{array}$ & $\begin{array}{l}\text { MEDIUM to } \\
\text { HIGH RISK }\end{array}$ \\
\hline 3 & $\begin{array}{l}\text { Inhibition of sterol } \\
\text { biosynthesis in } \\
\text { membranes }\end{array}$ & $\begin{array}{l}\text { Demethylation } \\
\text { inhibitors (DMI } \\
\text { fungicides) }\end{array}$ & $\begin{array}{l}\text { Imidazoles } \\
\text { Piperazine } \\
\text { Pyrimidines } \\
\text { Triazoles }\end{array}$ & $\begin{array}{l}\text { Imazalil } \\
\text { Triforine } \\
\text { Fenarimol } \\
\text { Difenoconazole } \\
\text { Fenbuconazole } \\
\text { Myclobutanil } \\
\text { Propiconazole } \\
\text { Triadimefon } \\
\end{array}$ & \\
\hline 4 & $\begin{array}{l}\text { Inhibition of nucleic } \\
\text { acid synthesis }\end{array}$ & Phenyl Amides & Acylalanines & $\begin{array}{l}\text { Metalaxyl } \\
\text { Metalaxyl-M }\end{array}$ & HIGH RISK \\
\hline 5 & $\begin{array}{l}\text { Inhibition of sterol } \\
\text { biosynthesis in } \\
\text { membranes }\end{array}$ & Amines (SBI: Class II) & Piperidines & Piperalin & \\
\hline 6 & $\begin{array}{l}\text { Inhibition of lipids and } \\
\text { membrane synthesis }\end{array}$ & $\begin{array}{l}\text { Phosphoro-thiolates, } \\
\text { dithiolanes }\end{array}$ & $\begin{array}{l}\text { None registered for } \\
\text { use in Florida }\end{array}$ & $\begin{array}{l}\text { None registered for use } \\
\text { in Florida }\end{array}$ & \\
\hline 7 & Inhibition of respiration & Carboxamides & & $\begin{array}{l}\text { Carboxin } \\
\text { Flutolanil } \\
\text { Oxycarboxin }\end{array}$ & \\
\hline 8 & $\begin{array}{l}\text { Inhibition of nucleic } \\
\text { acid synthesis }\end{array}$ & $\begin{array}{l}\text { Hydroxy-(2-amino) } \\
\text { pyrimidines }\end{array}$ & $\begin{array}{l}\text { None registered for } \\
\text { use in Florida }\end{array}$ & $\begin{array}{l}\text { None registered for use } \\
\text { in Florida }\end{array}$ & \\
\hline 9 & $\begin{array}{l}\text { Inhibition of amino } \\
\text { acids and protein } \\
\text { synthesis }\end{array}$ & Anilino-pyrimidines & & Cyprodinil & \\
\hline 10 & $\begin{array}{l}\text { Inhibition of mitosis } \\
\text { and cell division }\end{array}$ & $\mathrm{N}$-phenyl carbamates & $\begin{array}{l}\text { None registered for } \\
\text { use in Florida }\end{array}$ & $\begin{array}{l}\text { None registered for use } \\
\text { in Florida }\end{array}$ & \\
\hline 11 & Inhibition of respiration & $\begin{array}{l}\text { Quinone outside } \\
\text { inhibitors (Qol } \\
\text { fungicides) }\end{array}$ & $\begin{array}{l}\text { Methoxy-acrylates } \\
\text { Methoxy-carbamates } \\
\text { Oximino acetates } \\
\text { Oxazolidine-diones }\end{array}$ & $\begin{array}{l}\text { Azoxystrobin } \\
\text { Pyraclostrobin } \\
\text { Kresoxim methyl } \\
\text { Trifloxystrobin } \\
\text { Famoxadone }\end{array}$ & HIGH RISK \\
\hline 12 & $\begin{array}{l}\text { Inhibition of signal } \\
\text { transduction }\end{array}$ & $\begin{array}{l}\text { Phenyl Pyrroles (PP } \\
\text { fungicides) }\end{array}$ & & Fludioxonil & \\
\hline 13 & $\begin{array}{l}\text { Inhibition of signal } \\
\text { transduction }\end{array}$ & Quinolines & $\begin{array}{l}\text { None registered for } \\
\text { use in Florida }\end{array}$ & $\begin{array}{l}\text { None registered for use } \\
\text { in Florida }\end{array}$ & \\
\hline 14 & $\begin{array}{l}\text { Inhibition of lipids and } \\
\text { membrane synthesis }\end{array}$ & $\begin{array}{l}\text { Aromatic } \\
\text { hydrocarbons (AH } \\
\text { fungicides) }\end{array}$ & & $\begin{array}{l}\text { Chloroneb } \\
\text { Dicloran } \\
\text { PCNB }\end{array}$ & \\
\hline 15 & $\begin{array}{l}\text { Inhibition of glucan } \\
\text { and cell wall synthesis }\end{array}$ & Cinnamic acids & $\begin{array}{l}\text { None registered for } \\
\text { use in Florida }\end{array}$ & $\begin{array}{l}\text { None registered for use } \\
\text { in Florida }\end{array}$ & \\
\hline
\end{tabular}


Table 1. FRAC's classification of fungicides registered for use in Florida by FRAC numerical code, mode of action, chemical group, and active ingredient common name with high risk indication.

\begin{tabular}{|c|c|c|c|c|c|}
\hline $\begin{array}{l}\text { FRAC } \\
\text { code }^{*}\end{array}$ & Mode of action & Group name & Chemical group & Common name & $\begin{array}{l}\text { Resistance } \\
\text { risk } \\
\text { indication } \\
\end{array}$ \\
\hline 16.1 & $\begin{array}{l}\text { Inhibition of melanin } \\
\text { synthesis in cell wall }\end{array}$ & $\begin{array}{l}\text { Melanin biosynthesis } \\
\text { inhibitors - } \\
\text { reductase (MBI-R } \\
\text { fungicides) }\end{array}$ & $\begin{array}{l}\text { None registered for } \\
\text { use in Florida }\end{array}$ & $\begin{array}{l}\text { None registered for use } \\
\text { in Florida }\end{array}$ & \\
\hline 16.2 & $\begin{array}{l}\text { Inhibition of melanin } \\
\text { synthesis in cell wall }\end{array}$ & $\begin{array}{l}\text { Melanin biosynthesis } \\
\text { inhibitors - } \\
\text { dehydratase }\end{array}$ & $\begin{array}{l}\text { None registered for } \\
\text { use in Florida }\end{array}$ & $\begin{array}{l}\text { None registered for use } \\
\text { in Florida }\end{array}$ & \\
\hline 17 & $\begin{array}{l}\text { Inhibition of sterol } \\
\text { biosynthesis in } \\
\text { membranes }\end{array}$ & $\begin{array}{l}\text { Hydroxyanilides (SBI: } \\
\text { Class III) }\end{array}$ & & Fenhexamid & \\
\hline 18 & $\begin{array}{l}\text { Inhibition of sterol } \\
\text { biosynthesis in } \\
\text { membranes }\end{array}$ & (SBI: Class IV) & $\begin{array}{l}\text { None registered for } \\
\text { use in Florida }\end{array}$ & $\begin{array}{l}\text { None registered for use } \\
\text { in Florida }\end{array}$ & \\
\hline 19 & $\begin{array}{l}\text { Inhibition of glucan } \\
\text { and cell wall } \\
\text { synthesis }\end{array}$ & Polyoxins & $\begin{array}{l}\text { Peptidyl pyrimidine } \\
\text { nucleoside }\end{array}$ & Polyoxin & \\
\hline 20 & $\begin{array}{l}\text { Inhibition of mitosis } \\
\text { and cell division }\end{array}$ & Phenylureas & $\begin{array}{l}\text { None registered for } \\
\text { use in Florida }\end{array}$ & $\begin{array}{l}\text { None registered for use } \\
\text { in Florida }\end{array}$ & \\
\hline 21 & $\begin{array}{l}\text { Inhibition of } \\
\text { respiration }\end{array}$ & $\begin{array}{l}\text { Quinone inside } \\
\text { inhibitors (Qil } \\
\text { fungicides) }\end{array}$ & & Cyazofamid & $\begin{array}{l}\text { MEDIUM to } \\
\text { HIGH RISK }\end{array}$ \\
\hline 22 & $\begin{array}{l}\text { Inhibition of mitosis } \\
\text { and cell division }\end{array}$ & Benzamides & & Zoxamide & \\
\hline 23 & $\begin{array}{l}\text { Inhibition of amino } \\
\text { acids and protein } \\
\text { synthesis }\end{array}$ & $\begin{array}{l}\text { Enopyranuronic acid } \\
\text { antibiotic }\end{array}$ & $\begin{array}{l}\text { None registered for } \\
\text { use in Florida }\end{array}$ & $\begin{array}{l}\text { None registered for use } \\
\text { in Florida }\end{array}$ & \\
\hline 24 & $\begin{array}{l}\text { Inhibition of amino } \\
\text { acids and protein } \\
\text { synthesis }\end{array}$ & $\begin{array}{l}\text { Hexopyranosyl } \\
\text { antibiotic }\end{array}$ & $\begin{array}{l}\text { None registered for } \\
\text { use in Florida }\end{array}$ & $\begin{array}{l}\text { None registered for use } \\
\text { in Florida }\end{array}$ & \\
\hline 25 & $\begin{array}{l}\text { Inhibition of amino } \\
\text { acids and protein } \\
\text { synthesis }\end{array}$ & $\begin{array}{l}\text { Glucopyranosyl } \\
\text { antibiotic }\end{array}$ & & Streptomycin & HIGH RISK \\
\hline 26 & $\begin{array}{l}\text { Inhibition of glucan } \\
\text { and cell wall synthesis }\end{array}$ & $\begin{array}{l}\text { Glucopyranosyl } \\
\text { antibiotic }\end{array}$ & $\begin{array}{l}\text { None registered for } \\
\text { use in Florida }\end{array}$ & $\begin{array}{l}\text { None registered for use } \\
\text { in Florida }\end{array}$ & \\
\hline 27 & Unknown & $\begin{array}{l}\text { Cyanoacetamide- } \\
\text { oximes }\end{array}$ & & Cymoxanil & \\
\hline 28 & $\begin{array}{l}\text { Inhibition of lipids and } \\
\text { membrane synthesis }\end{array}$ & Carbamates & & Propamocarb & \\
\hline 29 & Inhibition of respiration & & 2,6-dinitroanilines & Fluazinam & \\
\hline 30 & Inhibition of respiration & $\begin{array}{l}\text { Organo tin } \\
\text { compounds }\end{array}$ & $\begin{array}{l}\text { Tri phenyl tin } \\
\text { compounds }\end{array}$ & Fentin hydroxide & \\
\hline 31 & $\begin{array}{l}\text { Inhibition of nucleic } \\
\text { acid synthesis }\end{array}$ & Carboxylic acids & $\begin{array}{l}\text { None registered for } \\
\text { use in Florida }\end{array}$ & $\begin{array}{l}\text { None registered for use } \\
\text { in Florida }\end{array}$ & \\
\hline 32 & $\begin{array}{l}\text { Inhibition of nucleic } \\
\text { acid synthesis }\end{array}$ & Heteroaromatics & Isothiazolones & Octhilinone & \\
\hline
\end{tabular}


Table 1. FRAC's classification of fungicides registered for use in Florida by FRAC numerical code, mode of action, chemical group, and active ingredient common name with high risk indication.

\begin{tabular}{|c|c|c|c|c|c|}
\hline $\begin{array}{l}\text { FRAC } \\
\text { code }^{*}\end{array}$ & Mode of action & Group name & Chemical group & Common name & $\begin{array}{l}\text { Resistance } \\
\text { risk } \\
\text { indication }\end{array}$ \\
\hline 33 & Unknown & Phosphonates & Ethyl phosphonates & $\begin{array}{l}\text { Fosetyl-Al } \\
\text { Phosphorous acid }\end{array}$ & \\
\hline 34 & Unknown & Pthalamic acids & $\begin{array}{l}\text { None registered for } \\
\text { use in Florida }\end{array}$ & $\begin{array}{l}\text { None registered for use } \\
\text { in Florida }\end{array}$ & \\
\hline 35 & Unknown & Benzotriazines & $\begin{array}{l}\text { None registered for } \\
\text { use in Florida }\end{array}$ & $\begin{array}{l}\text { None registered for use } \\
\text { in Florida }\end{array}$ & \\
\hline 36 & Unknown & $\begin{array}{l}\text { Benzene- } \\
\text { sulfonamides }\end{array}$ & $\begin{array}{l}\text { None registered for } \\
\text { use in Florida }\end{array}$ & $\begin{array}{l}\text { None registered for use } \\
\text { in Florida }\end{array}$ & \\
\hline 37 & Unknown & Pyridazinones & $\begin{array}{l}\text { None registered for } \\
\text { use in Florida }\end{array}$ & $\begin{array}{l}\text { None registered for use } \\
\text { in Florida }\end{array}$ & \\
\hline 38 & $\begin{array}{l}\text { ATP production } \\
\text { (proposed) }\end{array}$ & & $\begin{array}{l}\text { Thiophene- } \\
\text { carboxamides }\end{array}$ & $\begin{array}{l}\text { None registered for use } \\
\text { in Florida }\end{array}$ & \\
\hline 39 & $\begin{array}{l}\text { Complex I of } \\
\text { respiration } \\
\text { (proposed) }\end{array}$ & & Pyrimidinamides & $\begin{array}{l}\text { None registered for use } \\
\text { in Florida }\end{array}$ & \\
\hline 40 & $\begin{array}{l}\text { Phospholipid } \\
\text { biosynthesis and cell } \\
\text { wall deposition } \\
\text { (proposed) }\end{array}$ & $\begin{array}{l}\text { CAA-fungicides } \\
\text { (carboxylic acid } \\
\text { amides) }\end{array}$ & $\begin{array}{l}\text { None registered for } \\
\text { use in Florida }\end{array}$ & $\begin{array}{l}\text { None registered for use } \\
\text { in Florida }\end{array}$ & \\
\hline 41 & $\begin{array}{l}\text { Protein synthesis } \\
\text { attachment of } \\
\text { aminoacyl-tRNA to } \\
\text { ribosomal acceptor } \\
\text { (A) site }\end{array}$ & $\begin{array}{l}\text { Tetracycline } \\
\text { antibiotic }\end{array}$ & & Oxytetracyline & HIGH RISK \\
\hline 42 & Unknown & Thiocarbamate & $\begin{array}{l}\text { None registered for } \\
\text { use in Florida }\end{array}$ & $\begin{array}{l}\text { None registered for use } \\
\text { in Florida }\end{array}$ & \\
\hline$P$ & $\begin{array}{l}\text { Host plant defense } \\
\text { induction }\end{array}$ & $\begin{array}{l}\text { Salicylic acid } \\
\text { pathway P1 }\end{array}$ & Benzothiadazole (BTH) & Acibenzolar & \\
\hline$M$ & $\begin{array}{l}\text { Multi-site contact } \\
\text { activity }\end{array}$ & $\begin{array}{l} \\
\text { M4 } \\
\text { M5 } \\
\text { M6 } \\
\text { M7 } \\
\text { M8 } \\
\text { M9 }\end{array}$ & $\begin{array}{l}\text { Inorganics } \\
\text { Inorganic } \\
\text { Dithio-carbamates and } \\
\text { relatives } \\
\text { Phthalimides } \\
\text { Chloronitriles } \\
\text { Sulphamides } \\
\text { Guanidines } \\
\text { Triazines } \\
\text { Quinones } \\
\text { (anthraquinones) }\end{array}$ & $\begin{array}{l}\text { Copper (different salts) } \\
\text { Sulfur } \\
\text { Ferbam } \\
\text { Mancozeb } \\
\text { Maneb } \\
\text { Metiram } \\
\text { Thiram } \\
\text { Ziram } \\
\text { Captan } \\
\text { Folpet } \\
\text { Chlorothalonil } \\
\text { None registered for use } \\
\text { in Florida } \\
\text { Dodine } \\
\text { None registered for use } \\
\text { in Florida } \\
\text { None registered for use } \\
\text { in Florida }\end{array}$ & \\
\hline NC & Not classified & $\mathrm{NC}$ & $\begin{array}{l}\text { Quinones } \\
\text { (anthraquinones) } \\
\text { Diverse }\end{array}$ & $\begin{array}{l}\text { Oils } \\
\text { Potassium bicarbonate }\end{array}$ & \\
\hline
\end{tabular}


Table 2. Cross listing of active ingredient common names with trade products registered for use in Florida.

\begin{tabular}{|c|c|}
\hline Common name & Trade products $\AA_{\circledast}$ \\
\hline Acibenzolar & Actigard \\
\hline Azoxystrobin (HIGH RISK) & Abound, Amistar, Dynasty, Heritage, Protégé, Quadris, Quilt, Uniform \\
\hline Captan & $\begin{array}{l}\text { Agrox Premiere, Captan, Captec, Captivate, Fungitrol C, Kernel } \\
\text { Guard, Maxima HB, Rescue One, SA-50, TCl, Vitavax PC }\end{array}$ \\
\hline Carbendazim (HIGH RISK) & Mauget, Mergal, Polyphase, Rocima \\
\hline Carboxin & Allerax, Kickstart, Prevail, Vitavax \\
\hline Chloroneb & Catapult, Delta Coat, Teremec, Nu-Coat \\
\hline Chlorothalonil & $\begin{array}{l}\text { Applause, Banol C, Bravado, Bravo, Busan, Chloro Gold, Chloronil, } \\
\text { Chlorosel, Chlorostar, Chlorothalonil, Concorde, Consyst, Daconil, } \\
\text { Echo, Equus, Fungonil, Manicure, Maxcide, Nopcocide, Nucide, } \\
\text { Pathguard, Pro Tech, Quadris, Quali-Pro, Ridomil Gold, SA-50, } \\
\text { Spectro, Thor, Twosome }\end{array}$ \\
\hline Copper (carbonate) & Captain, Nautique, Wolman E \\
\hline Copper (chelates of copper citrate) & Algimycin, Bioguard, Bordeaux, Pool Time \\
\hline Copper (ethanolamine complex) & Various swimming pool treatments \\
\hline Copper (ethylenediamine complex) & Various aquatic herbicides \\
\hline Copper (hydroxide) & $\begin{array}{l}\text { Champ, Champion, Cobra Rod, Cu-Bor, Funguran OH, Junction, } \\
\text { Kocide, Kop-Hydroxide, Mankocide, Neptune, Nu-Cop, Ridomil Gold } \\
\text { Copper, Spin Out, }\end{array}$ \\
\hline Copper (I oxide) & Various anti-fouling paints \\
\hline Copper (II oxide) & Various wood preservatives \\
\hline Copper (metallic) & Various algaecides and anti-fouling paints \\
\hline Copper (naphthenate) & Various wood preservatives \\
\hline Copper (oxychloride) & Agra Cop, Coc, Kop Oxy 85, Microsperse \\
\hline $\begin{array}{l}\text { Copper (salts of fatty and rosin } \\
\text { acids) }\end{array}$ & Camelot, SA-50, Sunniland Coppercide, Tenn-Cop \\
\hline Copper (sulfate pentahydrate) & Various swimming pool treatments \\
\hline Copper (sulfate, anhydrous) & Copper-Z 4/4 \\
\hline Copper (triethanolamine complex) & Various algaecides and other water treatments \\
\hline $\begin{array}{l}\text { Cyazofamid (MEDIUM to HIGH } \\
\text { RISK) }\end{array}$ & Ranman \\
\hline Cymoxanil & Curzate, Tanos \\
\hline Cyprodinil & Switch, Vangard \\
\hline Dicloran & Botran \\
\hline Difenoconazole & Dividend, Incentive \\
\hline Dodine & Dodine, Elast, Syllit \\
\hline Famoxadone (HIGH RISK) & Tanos \\
\hline Fenarimol & Rubigan, Twosome \\
\hline Fenbuconazole & Enable, Indar \\
\hline Fenhexamid & Captevate, Decree, Elevate, \\
\hline Fentin hydroxide & Agri Tin, Enable, Orbit, Super Tin \\
\hline Ferbam & Ferbam \\
\hline Fluazinam & Omega \\
\hline Fludioxonil & $\begin{array}{l}\text { Apron, Dynasty, Graduate, Maxim, Medallion, Scholar, Switch, } \\
\text { Warden }\end{array}$ \\
\hline Flutolanil & Artisan, Contrast, Moncoat, Moncut, Prostar, Sysstar \\
\hline
\end{tabular}


Table 2. Cross listing of active ingredient common names with trade products registered for use in Florida.

\begin{tabular}{|c|c|}
\hline Common name & Trade products ${ }^{*} \circledast$ \\
\hline Folpet & Folpet, Fungitrol, several wood preservatives \\
\hline Fosetyl-Al & Aliette, Prodigy, Signature \\
\hline Imazalil & Clinafarm, Deccozil, Freshgard, Fungaflor, Magnate \\
\hline lodocarb & Many commercial preservative products available \\
\hline Iprodione & Iprodione, Lesco 18 Plus, Rovral, Sextant \\
\hline Kresoxim-methyl (HIGH RISK) & Cygnus, Sovran \\
\hline Mancozeb & $\begin{array}{l}\text { Acrobat MZ, Clean Crop Potato Seed, Cuprofix MZ, Dithane, Fore, } \\
\text { Gavel, Junction, Mancozide, Manhandle, Manzate, Maxim, Moncoat, } \\
\text { Nubark, Pentathlon, Penncozeb, Protect, Ridomil Gold MZ, Stature, } \\
\text { Tops MZ Gaucho, Ziban }\end{array}$ \\
\hline Maneb & Maneb, Manex, Pentathlon, Seed Treatment for Potatoes \\
\hline Mefenoxam (HIGH RISK) & $\begin{array}{l}\text { Agrox Premier, Allegiance, Allerax, Apron, Catapult, Delta Coat, } \\
\text { Dividend, Dynasty, Flouronil, Incentive, Maxim, Mefenoxam, } \\
\text { Meta-Mil, Prevail, Quell, Ridomil Gold, Subdue, System 3, Ultra } \\
\text { Flourish, Uniform }\end{array}$ \\
\hline Metiram & Polyram \\
\hline Myclobutanil & Eagle, Immunox, Laredo, Manhandle, Nova, Systhane, \\
\hline Octhilinone & $\begin{array}{l}\text { Arch, Dobercide, Kathon, Mergal, Milbrex, Rocima, Skane, Tex-Stat, } \\
\text { Thor }\end{array}$ \\
\hline Oils & Biozide, Decident \\
\hline Oxycarboxin & Plantivax, Provax \\
\hline Oxytetracylince (HIGH RISK) & Flameout, OTC, Star Brite \\
\hline Phosphorous acids & Magellan, Phostrol \\
\hline Piperalin & Pipron \\
\hline Polyoxin & Endorse \\
\hline Potassium bicarbonate & Agricure, Armicarb, Kaligreen \\
\hline Propamocarb & Banol, Previcur \\
\hline Propiconazole & $\begin{array}{l}\text { Alamo, Banner, Busan, Contend, Honor Guard, Infuse, Montero, } \\
\text { Orbit, Premier, Propensity, Quilt, Spectator, Stratego, Tilt }\end{array}$ \\
\hline Pyraclostrobin (HIGH RISK) & Cabrio, Headline, Insignia, Pristine \\
\hline PCNB & $\begin{array}{l}\text { Blocker, Defend, Parflo, Prevail, Revere, System 3, Terraclor, } \\
\text { Turfcide, Vitavax }\end{array}$ \\
\hline Streptomycin (HIGH RISK) & $\begin{array}{l}\text { Agri Mycin, Bac-Master, Firewall, Seed Treatment for Potatoes, } \\
\text { Streptrol }\end{array}$ \\
\hline Sulfur & Many commercial products \\
\hline Thiabendazole (HIGH RISK) & $\begin{array}{l}\text { Add-2, Decco Salt, Di-All, Fresh Ban, Fresh Mark, Freshgard, } \\
\text { Irgagard, Metasol, Post Harvest Lustr, Shield-Brite, Sta-Fresh, } \\
\text { Stay-Clean, Super Mildex }\end{array}$ \\
\hline Thiophanate-methyl (HIGH RISK) & Banrot, Cavalier, Fungo, Quali-Pro, Tee-Off, Topsin, \\
\hline Thiram & $\begin{array}{l}\text { Allerax, Bulb Saver, Defiant, Raxil, Spotrete, TCI Protector L, Thiram, } \\
\text { Vitavax }\end{array}$ \\
\hline Triadimefon & Armada, Bayleton, Fung Away, Fungisol, Fung-Onil, Strike \\
\hline Trifloxystrobin (HIGH RISK) & Armada, Compass, Flint, Gem, Twist, Stratego \\
\hline Triforine & Orthenex, Rosepride \\
\hline Vinclozolin & Curalan, Touche \\
\hline Ziram & Vancide, Ziram \\
\hline Zoxamide & Gavel \\
\hline
\end{tabular}


Table 2. Cross listing of active ingredient common names with trade products registered for use in Florida.

\begin{tabular}{||l|l||}
\hline \hline Common name & Trade products ${ }^{\circledR}$ \\
\hline${ }^{*}$ Trade product contains at least this sole active ingredient, but may be pre-mixed with additional active \\
ingredients. Consult product label ingredient statements.
\end{tabular}

